\title{
RND efflux pump systems in Acinetobacter, with special emphasis on their role in quorum sensing
}

\author{
Bindu Subhadra ${ }^{1}$, Man Hwan $\mathrm{Oh}^{2}$ and Chul Hee Choi ${ }^{1 *}$ \\ ${ }^{*}$ Department of Microbiology and Medical Science, Chungnam National University School of Medicine, Daejeon, \\ South Korea 35015 \\ ${ }^{2}$ Department of Nanobiomedical Science, Dankook University, Cheonan, South Korea 31116
}

\author{
Corresponding \\ Chul Hee Choi \\ Department of Microbiology and Medical \\ Science, Chungnam National University School \\ of Medicine, Daejeon, South Korea 35015 \\ Phone : $+82-42-580-8246$ \\ Fax : $+82-42-585-3686$ \\ E-mail : choich@cnu.ac.kr
}

Received : February 10, 2019

Revised : March 6, 2019

Accepted : March 7, 2019
Acinetobacteris an important opportunistic, multidrug resistant pathogen causing majority of nosocomial infections worldwide. The multidrug resistance is attributed by a plethora of efflux pumps and the overexpression of the same mediates export of antimicrobial agents. Quorum sensing (QS) is the cell-to-cell communication system in which bacteria produces specific signaling molecules which are transported out to the surrounding environment to communicate with other bacterial cells. It has been noticed that multidrug efflux pumps like resistance-nodulation-cell division (RND) efflux pumps play an important role in QS by exporting these signaling molecules. This review discusses various RND efflux pumps and the current understanding of the interrelationship of RND efflux pumps and QS in Acinetobacter spp. Studies demonstrate that RND efflux pumps could be considered as potential targets to block QS thereby reducing pathogenesis and antibiotic resistance. The known RND efflux pump-mediated quorum quenching strategies for Acinetobacter and other bacterial strains are discussed in detail. Finally, the prospective quorum quenching strategies targeting the transcriptional regulators of RND efflux pumps to inhibit multidrug efflux pumps are addressed.

Key Words: Acinetobacter, RND efflux pumps, quorum sensing, quorum quenching

\section{INTRODUCTION}

No potential conflict of interest relevant to this article was reported.

\footnotetext{
Copyright (C) 2019 Journal of Bacteriology and Virology

(OThis is an Open Access article distributed under the terms of the Creative Commons Attribution Non-Commercial License (http://creativecommons.org/ license/by-nc/3.0/)
}

Nowadays, the applicability of antibiotics is undermined by the evolution of multidrug resistant bacteria. Bacteria has developed various resistance strategies to fight against antibiotic stress and the most known ones are modification of the antibiotic by hydrolysis, acetylation or adenylation of the specific drugs, phosphorylation, modification of the antibiotic target by mutation or methylation, isolating the toxic compound by non-essential proteins in the cell or altering the membrane permeability of drugs (1). The pathogenic bacteria can transfer the resistant determinants to other organisms by horizontal gene transfer as many of these resistant gene elements are located on plasmids, transposons and integrons $(2,3)$. The first line of defense for many bacteria is to prevent the entry of toxic compounds via the cell membrane. Though the cell membrane of bacteria acts as an effective barrier to prevent the entry of many toxic compounds because of its amphipathic nature, the compounds may find their way into cells through pores and porins in the outer membrane (4). Thus, the downregulation of the expression of porins is an effective way to block the entry of toxic compounds into the cells (4). 
In addition, bacteria express a plethora of multidrug efflux pumps and the overexpression of these pumps alone is enough to cause multidrug resistance (MDR), without additional resistance factors (5).

Multidrug efflux pumps are classified in six families; major facilitator superfamily (MFS), small multidrug resistance family (SMR), ATP-binding cassette superfamily (ABC), multidrug and toxic compound extrusion family (MATE), proteobacterial antimicrobial compound extrusion family (PACE) and resistance-nodulation-division superfamily (RND) (5). The SMR, MATE and MFS families form the main efflux pump systems in Gram-positive bacteria, while RND efflux pumps are widely distributed in Gram-negative bacteria. Depending on the specific family the efflux pumps belong to, they can be either single component transporters or multicomponent systems with an inner membrane transporter, outer membrane channel and a periplasmic protein, such as RND efflux pumps (6). The RND pumps are majorly associated with clinically relevant antibiotic resistance, such as AcrAB-TolC efflux pump of Escherichia coli (E. coll) and Salmonella enterica serovar Typhimurium ( $S$. typhimurium) and MexAB-OprM of Pseudomonas aeruginosa ( $P$. aeruginosa) (7). Just like other RND efflux pumps, AcrAB-TolC efflux pumps are tripartite efflux pumps having three components, a transporter (efflux) protein, AcrB in the inner membrane, an outer membrane protein channel, TolC and a periplasmic accessory protein, AcrA (8). The RND efflux pumps are usually expressed at a basal expression level in bacteria which helps them to survive in the presence of toxic compounds (9). The AcrAB efflux pump is reported to recognize and transport a wide range of structurally unrelated compounds including antibiotics, bile salts, dyes and detergents (10). The substrate profile of AcrAB-TolC efflux pump of $E$. coli includes chloramphenicol, lipophilic $\beta$-lactams, fluoroquinolones, tetracycline, rifampin, novobiocin, fusidic acid, nalidixic acid, ethidium bromide, acriflavine, bile salts, short-chain fatty acids, SDS, Triton X-100, and triclosan (11-15).

Apart from their involvement in antibiotic resistance, RND efflux pumps are reported to play a role in bacterial pathogenicity by contributing to colonization and persistence of bacteria in their ecological niche (9). These efflux pumps extrude various host-derived antimicrobial compounds such as bile salts, fatty acids and detergents promoting the adaptation and survival of the bacterium in their ecological and physiological niches (16). The efflux of bile salts via AcrAB efflux pumps or its homologs has been reported in E. coli, P. aeruginosa, Neisseria gonorrhoeae (N. gonorrhoeae) and S. typhimurium (16-20). The defective mutations in these efflux pumps caused reduced virulence in several pathogens. In $S$. typhimurium, the inactivation of $a c r A B$ impaired intestinal colonization in murine model, indicating that the $A c r A B$ efflux pump is required for full virulence (21). In addition, Buckley et al. reported that $a c r B$ and to/C gene mutants colonize poorly in the avian gut, pointing that AcrAB-TolC system is essential for colonization of $S$. typhimurium in chickens (22). Similarly, In N. gonorrhoeae, a bacterial pathogen of the human genital mucosae, the deletion of $m t r D$ or $m \operatorname{tr} E$ gene, the product of which constitutes the MtrCDE efflux system lead to poor bacterial colonization in genito-urinary tract of female mice (23). The CmeABC efflux pump of Campylobacter jejuni confers resistance to a wide range of antimicrobials and the functional inhibition of this efflux pump could prevent bacterial host colonization (24). Recently, the role of AcrAB-TolC in virulence has also been reported in Klebsiella pneumoniae and Enterobacter cloacae in which the efflux pump defective mutants displayed reduced capability to infect mouse model $(25,26)$. In P. aeruginosa, the mutant lacking the MexAB-OprM, a homolog of AcrAB-TolC couldn't invade Madin-Darby canine kidney (MDCK) cells and it was suggested that the MexAB-OprM efflux system could export virulent determinants important for bacterial pathogenesis (27). The to/C mutant of $S$. typhimurium poorly adhered to both human embryonic intestinal cells and mouse monocyte macrophages, showing that the efflux pump system has a role in mediating bacterial adherence (22).

In addition to their direct role in bacterial pathogenesis, the efflux pumps affect bacterial virulence in a more indirect manner by altering the cell to cell communication (quorum sensing, QS) response in bacteria. QS is mediated by the release of chemical signaling molecules called autoinducers which are synthesized in vivo and needs to be transported across the cell membrane. The first report of autoinducers as substrate of RND family efflux pumps was made in studies of $P$. aeruginosa, in which the QS signals, acyl homoserine lactones (AHLs) are exported out by MexAB-OprM system (28, 29). P. aeruginosa overexpressing Mex pumps displayed reduced virulence due to the increased efflux of AHLs and the inability of the cells to accumulate QS signals. In addition, the overexpression of MexCD-OprJ and MexEF-OpRN are associated with the reduced expression of genes encoding type III secretion in $P$. aeruginosa (30). In E. coli, the overexpression of the QS regulator SdiA 
lead to the overexpression of AcrAB efflux pump, suggesting a potential role of this pump in QS (31). In this review, we focus on the RND efflux pump systems in the nosocomial pathogen, Acinetobacterspp. with special emphasis on their role in QS. In addition, we discuss the current state of knowledge on quorum quenching strategies by inhibiting the RND efflux pumps and future perspectives to effectively tackle this nosocomial pathogen.

\section{RND efflux pump systems in Acinetobacterspp}

In Acinetobacter baumannii (A. baumannil), three RND efflux systems, AdeABC (32), AdeFGH (33), and AdelJK (34) are reported to be primarily associated with MDR. AdeABC is the first characterized RND efflux pump in $A$. baumannii and is comprised of the major fusion protein AdeA, a multidrug transporter AdeB, and the outer membrane factor OMF (32). The AdeA and AdeB shares similarity to AcrA (55\%) and MexA (58\%) and to AcrB (68\%) and MexB (67\%), respectively, of $E$. coli and $P$. aeruginosa. The expression of $A d e A B C$ is under the tight control of the two-component regulatory system AdeR-AdeS, encoded by adeRS operon located upstream of ade $A B C$ operon (35). Ades is a sensor kinase which monitors the environmental conditions and activates or inactivates the response regulator, AdeR which regulates the expression of the efflux pump (35). In addition, the AdeABC and AdelJK RND pumps are indirectly regulated by the two-component regulatory system BaeSR in $A$. baumannii $(36,37)$. The AdeABC efflux system is not expressed in natural isolates of $A$. baumannii and the overexpression of the pump confers MDR by extruding aminoglycosides, $\beta$-lactams, fluoroquinolones, tetracyclines, tigecycline, macrolides, chloramphenicol, and trimethoprim (32). The AdeFGH efflux pump, encoded by the adeFGHoperon provides high-level resistance to fluoroquinolones, chloramphenicol, trimethoprim, and clindamycin and decreased susceptibility to tetracyclines, tigecycline, and sulfamethoxazole without affecting $\beta$-lactams and aminoglycosides (33). As in the case AdeABC efflux pump, AdeFGH pump is also not constitutively expressed in wild type strains. A putative LysR-type transcriptional regulator, named AdeL, encoded by adeL located upstream of the adeFGHoperon controls the expression of the AdeFGH efflux pump in $A$. baumannii (33). The AdelJK efflux pump, encoded by the adelJK operon is specific for the species $(38,39)$ where it confers intrinsic resistance to $\beta$-lactams, such as ticarcillin, cephalosporins, and aztreonam, fluoroquinolones, tetracyclines, tigecycline, lincosamides, rifampin, chloramphenicol, cotrimoxazole, novobiocin, and fusidic acid (34). It has been noticed that AdelJK acts in a synergistic fashion with AdeABC to export compounds such as tigecycline (34). The AdeXYZ efflux pump, encoded by the adeXYZ has $97 \%$ identity with AdelJK (34) and is found in Acinetobacter GDG3, Acinetobacter GDG13TU and Acinetobacter GDG 17 (40). However, the functional aspect of this efflux pump with respect to antimicrobial resistance has not well understood. Another efflux pump system, AdeDE confers resistance to aminoglycosides, fluoroquinolones, erythromycin, tetracycline and chloramphenicol in Acinetobacter spp. belonging to Acinetobacter genomic DNA group 3 (41). AdeDE is encoded by the membrane fusion protein gene, adeD and the RND transporter gene, adeE, and the outer membrane protein of AdeDE has not been identified.

\section{Quorum sensing in Acinetobacterspp}

Quorum sensing is the regulatory mechanism by which bacterial cells communicate each other producing signaling molecules called autoinducers. At a specific density in the environment, the autoinducers specifically bind to transcriptional regulators thereby altering the expression of various genes in a population (42). It has been known that QS plays a major role in the production of virulence factors, motility, nodulation, plasmid transfer, antibiotic production, bioemulsion production, bioluminescence and biofilm formation (43-45).

A. baumannii has one QS system which is mediated by the two-component system, Abal/AbaR which is homologous to the typical LuxI/LuxR system in E. coli. Abal is the autoinducer synthase that synthesizes AHLs, the signaling molecules which interact directly with the AbaR and this complex binds to specific promoter sequences (/ux-box) regulating the expression of QS target genes (46). It has been reported that Abal is responsible for the production of $\mathrm{N}$-(3-hydroxydodecanoyl)-L-HSL (3-hydroxy-C12-HSL) in $A$. baumannii strain M2 (46). The complete genome analysis of $A$. baumannii ATCC 17978 
suggested that the autoinducer synthase Abal and acyltransferase are the sole enzymes responsible for the synthesis of AHLS of varying chain lengths by this organism (46). The comparative analysis of the autoinducer synthase from Gram negative bacteria revealed that Abal is $45 \%$ identical to the autoinducer synthases from environemental isolates such as Halothiobacillus neapolitanus, Acidithiobacillus ferrooxidans ATCC 23270, Thiobacillus ferrooxidans, Pseudomonas spp. (pmr), Ralstonia solanacearum and Burkholderia ambifaria MC40-6 and 47\% identical to the one from Pseudomonas spp. RW10S (47). The communication among bacterial cells depending on the cell density plays a crucial role in the maturation of biofilms $(46,48)$. In $A$. baumannii, mutation in abal which is responsible for the production of AHLs lead to $30-40 \%$ reduction in biofilm formation compared to the parental strain (49). In addition, Niu et al. revealed that the exogenous addition of $\mathrm{AHLs}$ restored the biofilm maturation in abal mutant of $A$. baumannii M2 (46). In a different study, the supplementation of AHL exogenously produced biofilm in a biofilm-negative clinical isolate of Acinetobacter (50). Recently, a homologue of the Abal/AbaR system, referred as Anol/AnoR was characterized in $A$. nosocomialis (51). The Anol/AnoR system shares $94 \%$ identity with the Abal/AbaR of $A$. baumannii. The $A$. nosocomialis strain produces $\mathrm{N}$-(3-hydroxydodecanoyl)-L-homoserine lactone $(\mathrm{OH}-\mathrm{dDHL})$ as the signaling molecule and the ano/ mutant was not able to synthesize $\mathrm{OH}-\mathrm{dDHL}$ pointing that Anol is important for the production of AHLs (51). In addition, the expression of ano/ was derepressed in the anoR mutant, suggesting the role of AnoR as activator of anol in $A$. noscomialis. The deletion of anoR contributed to impaired biofilm formation and surface motility and the complementation of anoR in the anoR deletion mutant restored both the characteristics to that of wild type, indicating that AnoR is important for biofilm formation and motility (51).

It has been known that $63 \%$ of Acinetobacter strains identified so far produce more than one AHL (52). Though majority of the $A$. baumannii clinical isolates produce more than six AHLs, only one type is detected abundantly among them (50). Also, the $\mathrm{AHL}$ production is dependent on the culture conditions and four AHLs were identified when grown on minimal media and three when grown on minimal media with $0.1 \%$ tryptone in $A$. calcoaceticus BD413 (53). It is interesting to see that although multiple AHLs have been identified in Acinetobacter, only one autoinducer synthase is identified so far (46). Thus, it can be assumed that the AHL synthase has low specificity and it might be producing other AHLs as well. The quorum sensing signals are not homogenously distributed in Acinetobacter strains and thus it is difficult to distinguish the virulent and non-virulent strains based on the type of AHLs (52). However, one particular sensor, the Rf1-type sensor is widely distributed in strains belonging to $A$. calcoaceticus - A.baumannii complex (52).

\section{Interrelationship of RND efflux pumps and quorum sensing in Acinetobacterspp}

The QS system is mediated by the synthesis of signaling molecules and these molecules need to be exported into the surrounding media either by diffusion or active efflux (48). The AHLs vary in their carbon chain length and the short chain AHLs (4-8 carbon atoms) can easily diffuse through the cell membrane while the long chain AHLs (10-12 carbon atoms) need active transport across the membrane (48). The secretion of quorum sensing signals has been associated with multidrug efflux pumps $(54,55)$. In $A$. baumannii, the $\mathrm{AHLs}$ are exported into the extracellular environment through the AdeFGH efflux pump and the increase in concentration of AHLs in the extracellular environment accelerates the entry of AHLs into the intracellular environment to form AbaR-AHL complexes (54). The AHLs exported out are sensed by nearby $A$. baumannii cells and the increased interaction between cells through AHLs results in the acceleration of biofilm formation. In another study, the overexpression of AdeABC efflux pump in A. baumannii displayed increased biofilm formation and virulence phenotype though very little is known on various genes associated with mechanisms related to QS in this nosocomial pathogen (56). In a clinical isolate of $A$. baumanniistrain $\mathrm{S}$, the expression of multidrug efflux pump genes ade $A$ and $a d e B$ were induced by the production of AHLs (57). The loss of AHL production in the mutant strain lead to the decreased mRNA expression level of these efflux pump genes while the addition of AHLs restored their expression. Thus, it can be hypothesized that the AHL-mediated induction of AdeA and AdeB expression might be contributing to multidrug resistance in $A$. baumannii. Similarly, in a recent study, it was reported that the expression of acr $A$ and $a c r B$ efflux pump genes encoding the $A c r A B$ multidrug efflux system in $A$. nosocomialis is downregulated in the absence of the quorum 
sensing regulator, AnoR, suggesting that the synthesis of AHLs is important for the proper functioning of the $A c r A B$ efflux pump (58)

\section{RND efflux pump-mediated quorum quenching strategies}

Since QS allows bacteria to interact with the external environment and to adapt with the environmental changes thereby boosting its survival, inhibiting the QS system (quorum quenching) is a promising strategy to control infections (59-61). Multidrug efflux pumps have been reported to play a central role in the secretion of QS signals in Acinetobacterstrains apart from drug resistance and pathogenicity. It has been known that the AdeABC, AdeFGH and AcrAB efflux pumps are correlated to the QS system $(54,57,58)$ in Acinetobacter. In $A$. baumannii S strain, the AHLs promoted the expression of AdeA and AdeB genes in the presence of the antibiotic meropenem, suggesting that the strain produces AHLs to increase antibiotic resistance (57). In addition, the AdeFGH efflux pump of $A$. baumannii plays a crucial role in biofilm formation during infection by secreting AHLs (54). In A. nosocomialis, the deletion mutant of the transcriptional regulator, AcrR which negatively controls the expression of $A c r A B$ efflux pump displayed increased biofilm formation, motility and virulence (58). It was suggested that the enhanced expression of the AcrAB efflux pump in the acrR deletion mutant might be attributing well to the AHLs-mediated cell-to-cell signaling, contributing to increased virulence phenotypes. Thus, the development of inhibitors which target these multidrug efflux pumps would be a potential strategy to disrupt QS thereby controlling infections (Fig. 1). The already reported multidrug efflux pump inhibitors in A.baumannii include PA $\beta N$ (phenylalanine-arginine $\beta$-naphthylamide, also called MC- 207 110), CCCP (carbonyl cyanide-m-chlorophenylhydrazone), omeprazole, reserpine, verapamil, NMP(1-(1-naphthylmethyl)-piperazine), N-tert-butyl-2-(1-tert-butyltetrazol-5-yl) sulfanyl acetamide, (E)-4-((4-chlorobenzylidene)amino) benezenesulfonamide, etc. (62-66).

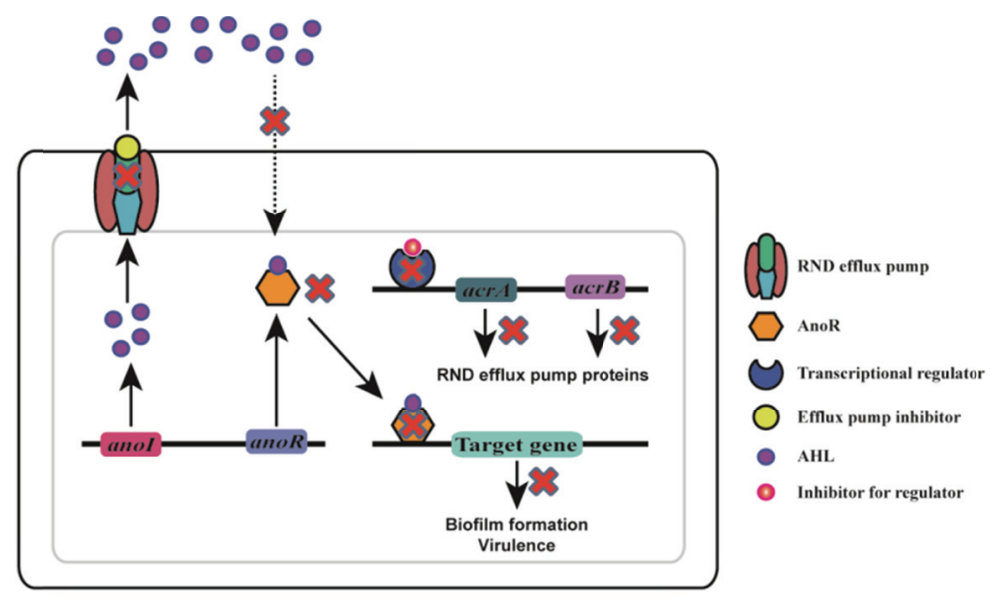

Figure 1. Schematic representation of quorum quenching strategies targeting multidrug effllux pumps, considering $A$. nosocomialis as a representative strain. ano/and anoR genes encode AHL signal synthase and AHL receptor/activator protein respectively. The efflux pump inhibitors binding to the efflux pumps inhibit the export of AHLs to the external environment blocking the quorum sensing system. The nearby bacterial cells are deprived of AHLs to be transported into the cells disrupting the formation of AnoR-AHL complex, thus preventing the binding of AnoR-AHL to the promoter regions of target genes including those for biofilm formation and virulence. The acr $A$ and $a c r B$ efflux pump genes are shown as examples of multidrug efflux pump genes in $A$. nosocomialis. The biomolecules which target the transcriptional regulators of efflux pump genes inhibit the expression of these genes leading to the disruption of the quorum sensing system. 
It has been reported that metallic nanoparticles represent a potential candidate to block multidrug efflux pumps in bacteria (67). In Staphylococcus aureus (S. aureus), zinc oxide nanoparticles displayed inhibitory role on efflux pumps (67). Similarly, the synergistic use of polyacrylic acid-coated iron oxide (magnetite) nanoparticles (PAA-MNP) with rifampicin against Mycobacterium smegmatis resulted in four-fold higher growth inhibition than that of rifampicin alone (68). Christena et al. reported the applicability of copper nanoparticles as efflux pump inhibitors in $S$. aureus and $P$. aeruginosa (69). The above studies prove the potential of nanoparticles as multidrug efflux pump inhibitors and the utility of these nanoparticles as efflux pump inhibitors could be investigated in Acinetobacterspp. as well. In addition, trifluoromethyl ketones (TFs) are reported to inhibit the QS response through their inhibition of efflux pumps in Chromobacterium violaceum 026 and E. coli (70). Since TFs inhibit efflux systems and QS, and also have significant antibacterial property, they could be exploited to treat infections that rely on QS and efflux-pump mediated multidrug-resistant phenotypes like Acinetobacter infections.

Apart from targeting the multidrug efflux pumps directly as a quorum quenching strategy, the efflux pump-mediated QS could also be inhibited indirectly by targeting the transctriptional regulators which controls the efflux pump expression (Fig. 1). In $A$. baumannii, the overexpression of the AdeABC efflux pump is mediated by the regulator AdeR (71). Also, the AdeABC and AdelJK pumps are positively regulated by the BaeSR regulon in $A$. baumannii (36). Thus, the modulators which target AdeR or BaeR would be a promising tool to regulate the expression of the corresponding multidrug efflux pumps in $A$. nosocomialis. In Yersinia enterocolitica, the AcrAB-TolC efflux pump is positively regulated by the regulator protein, OmpR (72). In our lab, in silico analysis and the electrophoretic mobility shift assay revealed that OmpR can bind to the acr $A B$ promoter region in $A$. nosocomialis, suggesting that OmpR might be controlling the expression of AcrAB efflux pump (data not published). However, further studies are of importance to elucidate the regulatory mechanism of OmpR in controlling the expression of AcrAB efflux pump in $A$. nosocomialis. In addition, screening for potential inhibitors of OmpR would be a desirable strategy to regulate the expression of OmpR thereby controlling the AcrAB efflux pump-mediated QS in $A$. nosocomialis.

\section{CONCLUSION}

Significant advances have been made in elucidating the functional aspects of various RND multidrug efflux pumps in Acinetobacter spp. RND efflux pumps are associated with MDR in Acinetobacter strains by extruding out antibiotics. Apart from this, these efflux pumps play an important role in the efflux of QS biomolecules thereby increasing bacterial virulence and biofilm formation. Thus, the inhibition of the RND efflux pumps would be a potential strategy to disrupt QS and to reduce the severity of infections. It is worth noticing that there are some promising RND efflux pump inhibitors like nanoparticles reported in other bacterial strains and the efficacy of the same could be tested in Acinetobacter as well. In addition, targeting the regulators of RND efflux pumps to inhibit multidrug efflux pumps would serve as a promising prospective quorum quenching strategy. Further studies are desired in this direction to completely understand the mechanism of transcriptional regulation of various efflux pumps and to screen the modulators of these regulators. The inhibition of RND efflux pumps not only increases the sensitity of bacterial cells to antimicrobial agents but also blocks the QS system thereby reducing pathogenesis. Thus, the studies which focus on screening new biomolecules targeting transcriptional regulators of multidrug efflux pumps would provide new therapeutic options to effectively control Acinetobacterinfections.

\section{ACKNOWLEDGMENT}

This work was supported by research fund of Chungnam National University (2017) 


\section{REFERENCES}

1) Walsh C. Molecular mechanisms that confer antibacterial drug resistance. Nature 2000;406:775-81.

2) Aarestrup FM. Veterinary drug usage and antimicrobial resistance in bacteria of animal origin. Basic Clin Pharmacol Toxicol 2005;96:271-81.

3) Witte W. Medical consequences of antibiotic use in agriculture. Science 1998;279:996-7.

4) Davin-Regli A, Bolla JM, James CE, Lavigne JP, Chevalier J, Garnotel E, et al. Membrane permeability and regulation of drug "influx and efflux" in enterobacterial pathogens. Curr Drug Targets 2008;9:750-9.

5) Blair JM, Richmond GE, Piddock LJ. Multidrug efflux pumps in Gram-negative bacteria and their role in antibiotic resistance. Future Microbiol 2014;9:1165-77.

6) Li XZ, Nikaido H. Efflux-mediated drug resistance in bacteria. Drugs 2004;64:159-204.

7) Piddock LJ. Clinically relevant chromosomally encoded multidrug resistance efflux pumps in bacteria. Clin Microbiol Rev 2006:19:382-402.

8) Koronakis V, Eswaran J, Hughes C. Structure and function of TolC: the bacterial exit duct for proteins and drugs. Annu Rev Biochem 2004;73:467-89.

9) Piddock LJ. Multidrug-resistance efflux pumps - not just for resistance. Nat Rev Microbiol 2006;4:629-36.

10) Nikaido H. Multidrug efflux pumps of gram-negative bacteria. J Bacteriol 1996;178:5853-9.

11) Folster JP, Shafer WM. Regulation of mtrF expression in Neisseria gonorrhoeae and its role in high-level antimicrobial resistance. J Bacteriol 2005;187:3713-20.

12) Nikaido H. Molecular basis of bacterial outer membrane permeability revisited. Microbiol Mol Biol Rev 2003;67:593-656.

13) Olliver A, Vallé M, Chaslus-Dancla E, Cloeckaert A. Role of an acrR mutation in multidrug resistance of in vitro-selected fluoroquinolone-resistant mutants of Salmonella enterica serovar Typhimurium. FEMS Microbiol Lett 2004:238:267-72.

14) Tegos G, Stermitz FR, Lomovskaya O, Lewis K. Multidrug pump inhibitors uncover remarkable activity of plant antimicrobials. Antimicrob Agents Chemother 2002;46:3133-41.

15) Wolter DJ, Smith-Moland E, Goering RV, Hanson ND, Lister PD. Multidrug resistance associated with mexXY expression in clinical isolates of Pseudomonas aeruginosa from a Texas hospital. Diagn Microbiol Infect Dis 2004;50:43-50.

16) Thanassi DG, Cheng LW, Nikaido H. Active efflux of bile salts by Escherichia coli. J Bacteriol 1997;179:2512-8.

17) Poole K, Krebes K, McNally C, Neshat S. Multiple antibiotic resistance in Pseudomonas aeruginosa: evidence for involvement of an efflux operon. J Bacteriol 1993;175:7363-72. 
18) Poole K, Gotoh N, Tsujimoto $H$, Zhao Q, Wada A, Yamasaki T, et al. Overexpression of the mexC-mexD-oprJ efflux operon in nfxB-type multidrug-resistant strains of Pseudomonas aeruginosa. Mol Microbiol 1996;21:713-24.

19) Piddock LJ, White DG, Gensberg K, Pumbwe L, Griggs DJ. Evidence for an efflux pump mediating multiple antibiotic resistance in Salmonella enterica serovar Typhimurium. Antimicrob Agents Chemother 2000;44:3118-21.

20) Shafer WM, Qu X, Waring AJ, Lehrer RI. Modulation of Neisseria gonorrhoeae susceptibility to vertebrate antibacterial peptides due to a member of the resistance/nodulation/division efflux pump family. Proc Natl Acad Sci U S A 1998;95:1829-33.

21) Lacroix FJ, Cloeckaert A, Grépinet O, Pinault C, Popoff MY, Waxin H, et al. Salmonella typhimurium acrB-like gene: identification and role in resistance to biliary salts and detergents and in murine infection. FEMS Microbiol Lett 1996:135:161-7.

22) Buckley AM, Webber MA, Cooles S, Randall LP, La Ragione RM, Woodward MJ, et al. The AcrAB-TolC efflux system of Salmonella enterica serovar Typhimurium plays a role in pathogenesis. Cell Microbiol 2006:8:847-56.

23) Jerse $A E$, Sharma ND, Simms AN, Crow ET, Snyder LA, Shafer WM. A gonococcal efflux pump system enhances bacterial survival in a female mouse model of genital tract infection. Infect Immun 2003;71:5576-82.

24) Lin J, Sahin O, Michel LO, Zhang Q. Critical role of multidrug efflux pump CmeABC in bile resistance and in vivo colonization of Campylobacter jejuni. Infect Immun 2003;71:4250-9.

25) Padilla E, Llobet E, Doménech-Sánchez A, Martínez-Martínez L, Bengoechea JA, Albertí S. Klebsiella pneumoniae AcrAB efflux pump contributes to antimicrobial resistance and virulence. Antimicrob Agents Chemother 2010;54:177-83.

26) Pérez A, Poza M, Fernández A, Fernández Mdel C, Mallo S, Merino M, et al. Involvement of the AcrAB-TolC efflux pump in the resistance, fitness, and virulence of Enterobacter cloacae. Antimicrob Agents Chemother 2012:56:2084-90.

27) Hirakata Y, Srikumar R, Poole K, Gotoh N, Suematsu T, Kohno S, et al. Multidrug efflux systems play an important role in the invasiveness of Pseudomonas aeruginosa. J Exp Med 2002:196:109-18.

28) Evans K, Passador L, Srikumar R, Tsang E, Nezezon J, Poole K. Influence of the MexAB-OprM multidrug efflux system on quorum sensing in Pseudomonas aeruginosa. J Bacteriol 1998;180:5443-7.

29) Poole K. Efflux-mediated antimicrobial resistance. J Antimicrob Chemother 2005;56:20-51.

30) Linares JF, López JA, Camafeita E, Albar JP, Rojo F, Martínez JL. Overexpression of the multidrug efflux pumps MexCD-OprJ and MexEF-OprN is associated with a reduction of type III secretion in Pseudomonas aeruginosa. J Bacteriol 2005;187:1384-91.

31) Rahmati S, Yang S, Davidson AL, Zechiedrich EL. Control of the AcrAB multidrug efflux pump by quorum-sensing regulator SdiA. Mol Microbiol 2002;43:677-85.

32) Magnet $S$, Courvalin $P$, Lambert T. Resistance-nodulation-cell division-type efflux pump involved in aminoglycoside resistance in Acinetobacter baumannii strain BM4454. Antimicrob Agents Chemother 2001;45:3375-80.

33) Coyne S, Rosenfeld N, Lambert T, Courvalin P, Périchon B. Overexpression of resistance-nodulation-cell division pump 
AdeFGH confers multidrug resistance in Acinetobacter baumannii. Antimicrob Agents Chemother 2010;54:4389-93.

34) Damier-Piolle L, Magnet S, Brémont S, Lambert T, Courvalin P. AdelJK, a resistance-nodulation-cell division pump effluxing multiple antibiotics in Acinetobacter baumannii. Antimicrob Agents Chemother 2008;52:557-62.

35) Marchand I, Damier-Piolle L, Courvalin P, Lambert T. Expression of the RND-type efflux pump AdeABC in Acinetobacter baumannii is regulated by the AdeRS two-component system. Antimicrob Agents Chemother 2004;48:3298-304.

36) Lin MF, Lin YY, Lan CY. The Role of the two-component system BaeSR in disposing chemicals through regulating transporter systems in Acinetobacter baumannii. PLoS One 2015;10:e0132843.

37) Lin MF, Lin YY, Yeh HW, Lan CY. Role of the BaeSR two-component system in the regulation of Acinetobacter baumannii ade $A B$ genes and its correlation with tigecycline susceptibility. BMC Microbiol 2014;14:119.

38) Lin L, Ling BD, Li XZ. Distribution of the multidrug efflux pump genes, ade $A B C$, adeDE and adelJK, and class 1 integron genes in multiple-antimicrobial-resistant clinical isolates of Acinetobacter baumanniF-Acinetobacter calcoaceticus complex. Int J Antimicrob Agents 2009;33:27-32.

39) Rajamohan G, Srinivasan VB, Gebreyes WA. Novel role of Acinetobacter baumannii RND efflux transporters in mediating decreased susceptibility to biocides. J Antimicrob Chemother 2010;65:228-32.

40) Chu YW, Chau SL, Houang ET. Presence of active efflux systems AdeABC, AdeDE and AdeXYZ in different Acinetobactergenomic DNA groups. J Med Microbiol 2006:55:477-8.

41) Chau SL, Chu YW, Houang ET. Novel resistance-nodulation-cell division efflux system AdeDE in Acinetobactergenomic DNA group 3. Antimicrob Agents Chemother 2004;48:4054-5.

42) Uroz S, Dessaux Y, Oger P. Quorum sensing and quorum quenching: the yin and yang of bacterial communication. ChemBioChem 2009;10:205-16.

43) Whitehead NA, Barnard AM, Slater H, Simpson NJ, Salmond GP. Quorum-sensing in Gram-negative bacteria. FEMS Microbiol Rev 2001;25:365-404.

44) Bassler BL. Small talk. Cell-to-cell communication in bacteria. Cell 2002;109:421-4.

45) Water CM, Bassler BL. Quorum sensing: cell-to-cell communication in bacteria. Annu Rev Cell Dev Biol 2005;21:319-46.

46) Niu C, Clemmer KM, Bonomo RA, Rather PN. Isolation and characterization of an autoinducer synthase from Acinetobacter baumannii. J Bacteriol 2008;190:3386-92.

47) Bhargava N, Sharma P, Capalash N. Quorum sensing in Acinetobacter. an emerging pathogen. Crit Rev Microbiol 2010;36:349-60.

48) Irie Y, Parsek MR. Quorum sensing and microbial biofilms. Curr Top Microbiol Immunol 2008;322:67-84.

49) Gaddy JA, Actis LA. Regulation of Acinetobacter baumannii biofilm formation. Future Microbiol 2009;4:273-8.

50) Prashanth K, Vasanth T, Saranathan R, Makki AR, Sudhakar P. Antibiotic resistance, biofilms and quorum sensing in 
Acinetobacter species. In: Dr. Marina P, editor. Antibiotic resistant bacteria - A coninuous challenge in the new millennium, IntechOpen; 2012.p.179-212.

51) Oh MH, Choi CH. Role of LuxIR Homologue AnolR in Acinetobacter nosocomialis and the Effect of Virstatin on the Expression of anoR Gene. J Microbiol Biotechnol 2015:25:1390-400.

52) González RH, Dijkshoorn L, Van den Barselaar M, Nudel C. Quorum sensing signal profile of Acinetobacterstrains from nosocomial and environmental sources. Rev Argent Microbiol 2009;41:73-8.

53) González RH, Nusblat A, Nudel BC. Detection and characterization of quorum sensing signal molecules in Acinetobacter strains. Microbiol Res 2001:155:271-7.

54) He X, Lu F, Yuan F, Jiang D, Zhao P, Zhu J, et al. Biofilm formation caused by clinical Acinetobacter baumannii isolates Is associated with overexpression of the AdeFGH efflux pump. Antimicrob Agents Chemother 2015;59:4817-25.

55) Kvist M, Hancock V, Klemm P. Inactivation of efflux pumps abolishes bacterial biofilm formation. Appl Environ Microbiol 2008;74:7376-82.

56) Richmond GE, Evans LP, Anderson MJ, Wand ME, Bonney LC, Ivens A, et al. The Acinetobacter baumannii two-component system AdeRS regulates genes required for multidrug efflux, biofilm formation, and virulence in a strain-specific manner. MBio 2016;7:e00430-16.

57) Dou Y, Song F, Guo F, Zhou Z, Zhu C, Xiang J, et al. Acinetobacter baumannii quorum-sensing signalling molecule induces the expression of drug-resistance genes. Mol Med Rep 2017:15:4061-8.

58) Subhadra B, Kim J, Kim DH, Woo K, Oh MH, Choi CH. Local repressor AcrR regulates AcrAB efflux pump required for biofilm formation and virulence in Acinetobacter nosocomialis. Front Cell Infect Microbiol 2018;8:270.

59) Chen G, Swem LR, Swem DL, Stauff DL, O'Loughlin CT, Jeffrey PD, et al. A strategy for antagonizing quorum sensing. Mol Cell 2011;42:199-209.

60) Kalia VC. Quorum sensing inhibitors: an overview. Biotechnol Adv 2013;31:224-45.

61) Roy $V$, Adams BL, Bentley WE. Developing next generation antimicrobials by intercepting Al-2 mediated quorum sensing. Enzyme Microb Technol 2011;49:113-23.

62) Pannek S, Higgins PG, Steinke P, Jonas D, Akova M, Bohnert JA, et al. Multidrug efflux inhibition in Acinetobacter baumannii. comparison between 1-(1-naphthylmethyl)-piperazine and phenyl-arginine-beta-naphthylamide. J Antimicrob Chemother 2006;57:970-4.

63) Ma Z, Cai SX, Tong WC, Ruan SC, Wang H. Role of the AdeABC efflux pump in carbapenems resistance of clinical isolates of Acinetobacter baumannii. Nan Fang Yi Ke Da Xue Xue Bao 2011:31:1378-81.

64) Kuo HY, Chang KC, Kuo JW, Yueh HW, Liou ML. Imipenem: a potent inducer of multidrug resistance in Acinetobacter baumannii. Int J Antimicrob Agents 2012;39:33-8.

65) Ribera A, Ruiz J, Jiminez de Anta MT, Vila J. Effect of an efflux pump inhibitor on the MIC of nalidixic acid for Acinetobacter baumannii and Stenotrophomonas maltophilia clinical isolates. J Antimicrob Chemother 
2002;49:697-8.

66) Mullié C, Guiheneuf R, Serra C, Tir Touil-Meddah A, Sonnet P. Efflux pumps in Acinetobacter baumannii. role in antibiotic resistance and interest of efflux pump inhibitors as additional therapeutic weapons. In: Méndez-Vilas A, editor. Microbiology Book Series \#6: "Antimicrobial research: Novel bioknowledge and educational programs". Formatex Research Center; 2017. p. 572-83.

67) Banoee M, Seif S, Nazari ZE, Jafari-Fesharaki P, Shahverdi HR, Moballegh A, et al. ZnO nanoparticles enhanced antibacterial activity of ciprofloxacin against Staphylococcus aureus and Escherichia coli. J Biomed Mater Res B Appl Biomater 2010:93:557-61.

68) Padwal P, Bandyopadhyaya R, Mehra S. Polyacrylic acid-coated iron oxide nanoparticles for targeting drug resistance in mycobacteria. Langmuir 2014;30:15266-76.

69) Christena LR, Mangalgowri V, Pradheeba P, Ahmed KB, Shalini BI, Vidyalakshmi M, et al. Copper nanoparticles as an efflux pump inhibitor to tackle drug resistant bacteria. RCS Adv 2015;5:12899-909.

70) Varga ZG, Armada A, Cerca P, Amaral L, Mior Ahmad Subki MA, Savka MA, et al. Inhibition of quorum sensing and efflux pump system by trifluoromethyl ketone proton pump inhibitors. In Vivo 2012:26:277-85.

71) Chang TY, Huang BJ, Sun JR, Perng CL, Chan MC, Yu CP, et al. AdeR protein regulates adeABC expression by binding to a direct-repeat motif in the intercistronic spacer. Microbiol Res 2016;183:60-7.

72) Raczkowska A, Trzos J, Lewandowska O, Nieckarz M, Brzostek K. Expression of the AcrAB components of the AcrAB-TolC multidrug efflux pump of Yersinia enterocolitica is subject to dual regulation by OmpR. PLoS One 2015;10:e0124248. 